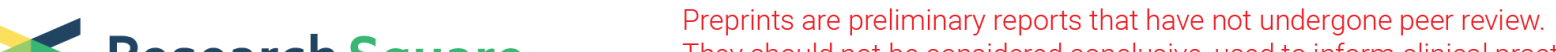 Research Square They should not be considered conclusive, used to inform clinical practice, or referenced by the media as validated information.
}

\section{Clinical characteristics and current treatment of critically ill patients with COVID-19 outside Wuhan, China: a multicenter, retrospective, observational study}

\section{Yuhong Chen}

Hebei Medical University Fourth Affiliated Hospital and Hebei Provincial Tumor Hospital

\section{Kun Zhang}

Hebei Medical University Fourth Affiliated Hospital and Hebei Provincial Tumor Hospital

\section{Guijun Zhu}

Hebei Medical University Fourth Affiliated Hospital and Hebei Provincial Tumor Hospital

\section{Lixia Liu}

Hebei Medical University Fourth Affiliated Hospital and Hebei Provincial Tumor Hospital

\section{Xixin Yan}

Second Hospital of Hebei Medical University

\section{Zhigang Cai}

Second Hospital of Hebei Medical University

\section{Zhongheng Zhang}

Zhejiang University School of Medicine Sir Run Run Shaw Hospital

Zhengjie Hu ( $\nabla$ syicu@vip.sina.com )

Hebei Medical University Fourth Affiliated Hospital and Hebei Provincial Tumor Hospital https://orcid.org/0000-0003-1404-5691

\section{Research}

Keywords: COVID-19, 2019 novel coronavirus, 2019-nCoV, SARS-CoV-2, critical ill patients

Posted Date: March 17th, 2020

DOl: https://doi.org/10.21203/rs.3.rs-17390/v1

License: (a) (i) This work is licensed under a Creative Commons Attribution 4.0 International License. Read Full License 


\section{Abstract}

Background: A novel coronavirus disease 2019 (COVID-19) occurred in Wuhan and rapidly spread elsewhere. The clinical characteristics and treatment of critical ill patients outside Wuhan remain unknown. We aimed to describe the epidemiology and treatment of critical ill patients with COVID-19 in Hebei province.

Methods: All patients were from designated hospitals of Hebei province and fit the criteria. We collected the clinical data, laboratory examinations and treatment of all participants.

Results: By 14 February 2020, 37 critical ill patients were included. The mean age of the patients was 58.73 (SD 13.76) years, 21 (56.80\%) patients were men, 18 (48.60\%) were familial cluster, 26 (70.30\%) patients had chronic illness. The patients with critical type had a longer period of confirmation time, more severe inflammation and lung injury, a lower lymphocyte percentage. All patients were treated with antiviral agents, $33(89.20 \%)$ with antibacterial agents, 35 (95.60\%) patients with methylprednisolone and traditional Chinese drugs. Nine $(60.00 \%)$ patients with critical type were treated with invasive mechanical ventilation, $9(60.00 \%)$ of those with complications. The patients with critical type received more fluid and more diuretics.

Conclusion: Patients with underlying disease and the confirmation time> 10 days were more likely to develop to critical type. The critical type patients had higher risk of infection, respiratory depression, circulatory collapse, and complications. The strategy of lung-protective mechanical ventilation and restrictive fluid management should be strictly followed.

\section{Background}

In December 2019, a cluster of pneumonia cases occurred in Wuhan and rapidly spread elsewhere in China and to the other Countries, eventually, this pneumonia was defined as an infectious disease and named novel coronavirus disease 2019 (COVID-19) [1, 2]. Up to 24 February 2020, 79, 370 people have been infected and 2,619 (3.30\%) patients died,including the data from other 29 countries. According to the Guidelines for Diagnosis and Treatment of Pneumonia Caused by 2019-nCoV (version 5) [3]区COVID-19 can be classified as mild, severe and critical types. Early identification and efficient treatment are of similar importance for critical patients with COVID-19.

Most literature focused on the epidemiological and clinical characteristics of general patients in Wuhan, only two studies outside Wuhan were available. One study suggested that the symptoms of patients in Zhejiang province were relatively mild than Hubei province [4]. Another study investigated the clinical course and outcomes of critically ill patients with COVID-19 in Wuhan. They found the mortality of those patients was up to $65 \%$, which may be associated with the mismatch between critical ill patients and critical care resources [5]. The clinical characteristics and treatment of critical ill patients outside Wuhan remain unknown. We aimed to describe the epidemiology clinical characteristics and treatment of critical ill patients infected with COVID-19 in Hebei province. 


\section{Methods}

\section{Study design and population}

A multicentre, retrospective, observational trail was conducted between 22 January 2020 to 14 February 2020 in 13 hospitals of Hebei province (Hebei, China), which were designated hospitals to treat patients with COVID-19. Inclusion criteria: 1. Diagnosed of COVID-19 by laboratory confirmation and the local health authority; 2 . Fit the criteria of severe type or critical type. Severe type was defined as respiratory distress, or respiratory rate $₫ 30$ breaths per minute, or percutaneous oxygen saturation $\left(\mathrm{SpO}_{2}\right)$ less than 93\% under resting conditions, or partial pressure of oxygen $\left(\mathrm{PaO}_{2}\right)$ /fraction of inspired oxygen $\left(\mathrm{FiO}_{2}\right) \leq$ $300 \mathrm{mmHg}(1 \mathrm{mmHg}=0.133 \mathrm{KPa})$. Critical type was defined as respiratory failure and required mechanical ventilation, or shock, or combined with other organ failure and required ICU monitoring/treatment. Exclusion criteria: 1. Pregnancy; 2 . Hospital length of stay $\leq 48$ hours; 3 . Age $\leq 18$ years.

\section{Data Collection}

Clinical medical records, nursing records, laboratory findings, and radiological examinations were collected. Any missing or uncertain records were obtained and clarified through direct communication with the managing physician.

We collected data on age, sex, exposure history, chronic medical histories (hypertension, cardiovascular disease, diabetes, pulmonary disease, cerebrovascular disease, liver disease, kidney Disease, malignancy, surgical history and smoking), symptoms from onset to hospital admission (fever, cough, expectoration, dyspnoea, fatigue, diarrhea, myalgia and headache), vital signs at confirmation of disease type [temperature, heart rate, systolic pressure,diastolic pressure, mean arterial pressure (MAP), respiratory rate (RR) and $\mathrm{SpO}_{2}$ ], chest computed tomography (CT) findings, laboratory findings at confirmation of disease type $\left[\mathrm{PH}, \mathrm{PO}_{2}\right.$, partial pressure of carbon dioxide $\left(\mathrm{PCO}_{2}\right), \mathrm{FiO}_{2}, \mathrm{PO}_{2} / \mathrm{FiO}_{2}(\mathrm{P} / \mathrm{F})$, Lactate concentration (Lac) ,white blood cell count(WBC), neutrophil percentage (NEUT), lymphocyte percentage (LYMPH), lymphocyte count (LY\#), platelet count (PLT), C-reactive protein (CRP), procalcitonin (PCT), erythrocyte sedimentation rate (ESR), prothrombin time (PT), activated partial thromboplastin time (APTT), troponin, creatine kinase (CK), NTpro-BNP, blood urea nitrogen (BUN), serum creatinine concentration (Cr), Cystatin C, direct bilirubin (DBIL), indirect bilirubin (IBIL), albumin, sodium ( $\mathrm{Na}$ ), potassium (K),blood glucose (Glu), CD 4cell

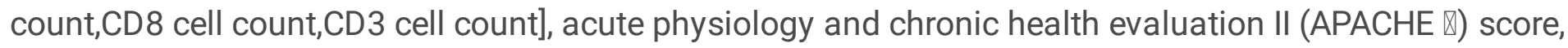
sequential organ failure assessment (SOFA) score, treatment [antiviral agent, antibacterial agents, antifungal agents, corticosteroids, gamma globulin, thymalfasin, human albumin, blood products, Traditional Chinese Drugs, Xuebijing injection, lyophilized recombinant human brain natriuretic peptide (rhBNP), Levosimendan, vasoactive agent, sedatives, analgesics, neuromuscular blocking agents, diuretics, the situation of fluid intake and output] and comorbidities (cardiac injury, acute kidney injury, gastrointestinal bleeding and liver dysfunction). Most mechanical ventilator settings and recordings were not recorded. 


\section{Statistical analysis}

All analyses were performed with SPSS for Windows version 22.0. Descriptive data with normal distribution were expressed as mean with standard deviation (SD). T-test was used for group comparison. Analysis of variance was employed for comparisons of multiple groups. Skewed quantitative data were expressed as medians and interquartile ranges $[\mathrm{M}(\mathrm{QL}, \mathrm{QU})]$, and used nonparametric rank sum test [6]. Pearson chi-square test or Fisher exact probability test was used to compare categorical variables. $\mathrm{P}<0.05$ were considered statistically significant.

\section{Results}

\section{Epidemiological characteristics}

By 14 February 2020, 291 patients with COVID-19 have been diagnosed in Hebei province, including 44 $(15.12 \%)$ critical ill patients. Of these, 7 patients were ineligible and 37 patients were included for efficacy analyses (Fig. 1). The mean age was 58.73 years (SD 13.76), and 21 (56.80\%) patients were men. No participant had a history of exposure to the Huanan seafood market, and $83.78 \%$ patients could provide a history of contact, nearly half (48.60\%) of the patients were familial cluster (Table 1). Twenty-six (70.30\%) patients had chronic illness: 17 (45.90\%) had hypertension, 9 (24.30\%) had cardiovascular disease, 9 $(24.30 \%)$ had diabetes, 7 (18.90\%) had pulmonary disease. A few patients had cerebrovascular disease, liver or kidney disease, and malignancy. Twelve (32.40\%) had history of surgery, and only $3(8.10 \%)$ had smoking history (Table 1). 
Table 1

Demographics and baseline characteristics of critical ill patients with COVID-19

\begin{tabular}{|c|c|c|c|c|}
\hline & $\begin{array}{l}\text { Critical type }(n= \\
15)\end{array}$ & $\begin{array}{l}\text { Severe type }(n= \\
\text { 22) }\end{array}$ & $\begin{array}{l}\text { All patients }(n= \\
\text { 37) }\end{array}$ & $\mathbf{P}$ \\
\hline Sex(male) & $7(46.70 \%)$ & $14(63.60 \%)$ & $21(56.80 \%)$ & 0.31 \\
\hline Age(years) & $58.93(14.33)$ & $58.59(13.70)$ & $58.73(13.76)$ & 0.94 \\
\hline Exposure & $13(86.67 \%)$ & $18(81.82 \%)$ & $31(83.78 \%)$ & 0.69 \\
\hline $\begin{array}{l}\text { Exposure to Huanan seafood } \\
\text { market }\end{array}$ & 0 & 0 & 0 & \\
\hline Exposure to Wuhan people & $2(13.30 \%)$ & $4(18.20 \%)$ & $6(16.20 \%)$ & 0.69 \\
\hline Sojourn in Wuhan & $3(20.00 \%)$ & $7(31.80 \%)$ & $10(27.00 \%)$ & 0.43 \\
\hline Exposure to patients $\square$ & $7(46.70 \%)$ & $7(31.80 \%)$ & $14(37.80 \%)$ & 0.36 \\
\hline Familial cluster & $7(46.70 \%)$ & $11(50.00 \%)$ & $18(48.60 \%)$ & 0.84 \\
\hline Chronic illness & $13(86.70 \%)$ & $13(59.10 \%)$ & $26(70.30 \%)$ & 0.07 \\
\hline hypertension & $10(66.70 \%)$ & $7(31.80 \%)$ & $17(45.90 \%)$ & 0.04 \\
\hline Cardiovascular disease & $7(46.70 \%)$ & $2(9.10 \%)$ & $9(24.30 \%)$ & 0.01 \\
\hline Diabetes & $4(26.70 \%)$ & $5(22.70 \%)$ & $9(24.30 \%)$ & 0.78 \\
\hline pulmonary disease & $4(26.70 \%)$ & $3(13.60 \%)$ & $7(18.90 \%)$ & 0.32 \\
\hline Cerebrovascular disease & $2(13.30 \%)$ & $1(4.50 \%)$ & $3(8.10 \%)$ & 0.34 \\
\hline Liver disease & $2(13.30 \%)$ & $1(4.50 \%)$ & $3(8.10 \%)$ & 0.34 \\
\hline Kidney Disease & $2(13.30 \%)$ & 0 & $2(5.40 \%)$ & 0.08 \\
\hline Malignancy & $1(6.70 \%)$ & 0 & $1(2.70 \%)$ & 0.22 \\
\hline Surgery history & $5(33.30 \%)$ & $7(31.80 \%)$ & $12(32.40 \%)$ & 0.92 \\
\hline Smoking & $2(13.30 \%)$ & $1(4.50 \%)$ & $3(8.10 \%)$ & 0.34 \\
\hline \multicolumn{5}{|l|}{ Data are $\mathrm{n}(\%)$ or mean (SD). } \\
\hline QPatients who have confirmed & -19 infection or & & eing infected. & \\
\hline
\end{tabular}

\section{Symptoms And Chest Radiography Findings}

The most common symptoms were fever (97.30\%), cough (70.30\%), expectoration (43.2\%) and dyspnea (37.80\%), fatigue (27.00\%), myalgia (16.20\%), diarrhea (10.80\%) and headache $(8.10 \%)$ occurred rarely (Table 2). The average time from onset of symptoms to laboratory confirmation was 6.61 (SD 4.49) days. 
The patients with critical type had a longer period of confirmation time, 8 of 15 (53.33\%) patients lasted for more than 10 days, which observed only in 2 of 22 (9.09\%) severe type patients (Table 2). All but two patients $(94.60 \%)$ with severe type had bilateral infiltrates on chest CT, critical type demonstrated diffuse, extensive interstitial and alveolar infiltrates, while most of patients with severe type showed localized infiltrates (Fig. 2), So critical type patients were more likely to develop shortness of breath (Table 2).

Table 2

Symptoms and chest radiography findings of critical ill patients with COVID-19

\begin{tabular}{|lllll|}
\hline & $\begin{array}{l}\text { Critical type(n } \\
\text { (15) }\end{array}$ & $\begin{array}{l}\text { Severe type(n= } \\
\text { 22) }\end{array}$ & $\begin{array}{l}\text { All patients(n= } \\
\mathbf{3} \text { ( })\end{array}$ \\
\hline Fever & $15(100.00 \%)$ & $21(95.5 \%)$ & $36(97.30 \%)$ & 0.40 \\
\hline Cough & $13(86.70 \%)$ & $13(59.10 \%)$ & $26(70.30 \%)$ & 0.07 \\
\hline Expectoration & $6(40.00 \%)$ & $10(45.50 \%)$ & $16(43.20 \%)$ & 0.74 \\
\hline Dyspnea & $9(60.00 \%)$ & $5(22.70 \%)$ & $14(37.80 \%)$ & 0.02 \\
\hline Fatigue & $3(20.00 \%)$ & $7(31.80 \%)$ & $10(27.00 \%)$ & 0.43 \\
\hline Myalgia & $1(6.70 \%)$ & $5(22.70 \%)$ & $6(16.20 \%)$ & 0.19 \\
\hline Diarrhea & $1(6.70 \%)$ & $3(13.60 \%)$ & $4(10.80 \%)$ & 0.50 \\
\hline Headache & $1(6.70 \%)$ & $2(9.10 \%)$ & $3(8.10 \%)$ & 0.79 \\
\hline $\begin{array}{l}\text { Time from illness onset to } \\
\text { confirmation (days) }\end{array}$ & $8.33(5.11)$ & $5.38(3.63)$ & $6.61(4.49)$ & 0.07 \\
\hline Confirmation time $\geq 10 d$ & $8(53.30 \%)$ & $2(9.10 \%)$ & $10(27.00 \%)$ & 0.00 \\
\hline $\begin{array}{l}\text { Bilateral involvement on chest } \\
\text { radiographs }\end{array}$ & $15(100.00 \%)$ & $20(90.90 \%)$ & $35(94.60 \%)$ & 0.23 \\
\hline Ground-glass opacities & $15(100.00 \%)$ & $15(68.20 \%)$ & $30(81.10 \%)$ & 0.02 \\
\hline Consolidation & $6(40.00 \%)$ & $7(31.80 \%)$ & $13(35.10 \%)$ & 0.61 \\
\hline Reticular pattern & $11(73.30 \%)$ & $2(9.10 \%)$ & $13(35.10 \%)$ & 0.00 \\
\hline Thick interlobular septa & $3(20.00 \%)$ & $2(9.10 \%)$ & $5(13.50 \%)$ & 0.34 \\
\hline Pleural effusion & $2(13.30 \%)$ & $1(4.50 \%)$ & $3(8.10 \%)$ & 0.34 \\
\hline Lung edema & $5(33.30 \%)$ & 0 & $5(13.50 \%)$ & 0.00 \\
\hline Data are n (\%) or mean (SD). & & & & \\
\hline
\end{tabular}

\section{Vital Signs And Laboratory Findings}


We compared the vital signs and laboratory findings between the two groups at the confirmation of disease type (Table 3). The highest daily temperature, heart rate and respiratory rate were higher in the critical group. Vasoconstrictive agents were used in $9(60.00 \%)$ patients with critical type, because they had a lower blood pressure (Table 3 and Table 4). The oxygenation index was decreased significantly in critical group $\left(\varangle 100 \mathrm{mmHg}\right.$ ), so critical type patients must be treated with higher $\mathrm{FiO}_{2}$ to maintain $\mathrm{SpO}_{2} \geq$ 93\% (Table 3), while most patients with severe group had better oxygenation index ( $\otimes 100 \mathrm{mmHg}$ ). Both groups had a mild elevation of Lac, but there was no significant difference. In the critical group, the levels of WBC, NEUT, CRP, PCT and ESR were markedly elevated, conversely, the level of lymphocyte percentage was lower (Table 3). Additionally, patients with critical type had mild elevation of creatine kinase. Biomarkers of coagulation, liver and kidney function, electrolytes were within normal range. Patients with critical type had higher APACHE II and SOFA scores. The level of CD4, CD8, CD3 and CD4/CD 8 were measured in five patients with critical type and four patients with severe type. There was a trend showing $T$ cell subsets were lower level in critical type (Supplemental Table 1). 
Table 3

Vital signs and laboratory findings of critical ill patients with COVID-19

\begin{tabular}{|c|c|c|c|}
\hline & Critical type(n=15) & Severe type(n=22) & $\mathbf{P}$ \\
\hline Temperature $\left({ }^{\circ} \mathrm{C}\right)$ & $38.54(0.63)$ & $37.24(0.75)$ & 0.00 \\
\hline Heart rate (beats per min) & $97(26)$ & 81(13) & 0.04 \\
\hline Systolic pressures (mmHg) & $111(24)$ & $130(14)$ & 0.01 \\
\hline Diastolic pressure (mmHg) & 69(14) & 78(13) & 0.04 \\
\hline MAP (mmHg) & $82(17)$ & $95(12)$ & 0.01 \\
\hline RR (breaths per min) & $25.00(22.00,38.00)$ & $20.00(18.00,22.00)$ & 0.00 \\
\hline $\mathrm{SpO}_{2}(\%)$ & $93.00(92.00,98.00)$ & $95.00(92.75,96.25)$ & 0.63 \\
\hline $\mathrm{PH}$ & $7.46(0.05)$ & $7.43(0.04)$ & 0.04 \\
\hline $\mathrm{PO}_{2}(\mathrm{mmHg})$ & $81.20(61.60,87.00)$ & $75.4(64.00,86.70)$ & 0.66 \\
\hline $\mathrm{PCO}_{2}(\mathrm{mmHg})$ & $31.77(6.01)$ & $36.37(4.55)$ & 0.01 \\
\hline $\mathrm{FiO}_{2}(\%)$ & 74.47(19.05) & $33.91(8.52)$ & 0.00 \\
\hline $\mathrm{P} / \mathrm{F}(\mathrm{mmHg})$ & $95.60(83.30,161.00)$ & $231.00(182.00,279.00)$ & 0.00 \\
\hline Lac (mmol/L) & $2.40(1.58,4.70)$ & $2.10(1.45,2.98)$ & 0.35 \\
\hline $\operatorname{WBC}\left(\times 10^{9} / \mathrm{L}\right)$ & $11.97(6.58)$ & $6.37(3.64)$ & 0.01 \\
\hline NEUT (\%) & $89.05(6.11)$ & 75.58(17.09) & 0.00 \\
\hline LYMPH (\%) & $8.16(5.22)$ & $16.30(14.75)$ & 0.02 \\
\hline $\mathrm{LY} \#\left(\times 10^{9} / \mathrm{L}\right)$ & $0.67(0.43,0.73)$ & $0.69(0.38,0.99)$ & 0.72 \\
\hline $\operatorname{PLT}\left(\times 10^{9} / \mathrm{L}\right)$ & $143.00(107.00,283.00)$ & $183.00(144.00,204.50)$ & 0.49 \\
\hline CRP (mg/L) & $82.50(52.00,217.30)$ & $22.94(14.43,53.59)$ & 0.00 \\
\hline PCT (ng/mL) & $0.47(0.17,1.21)$ & $0.10(0.05,0.22)$ & 0.01 \\
\hline \multicolumn{4}{|c|}{ Data are median(first quartile,third quartile)or mean(SD). } \\
\hline \multicolumn{4}{|c|}{ 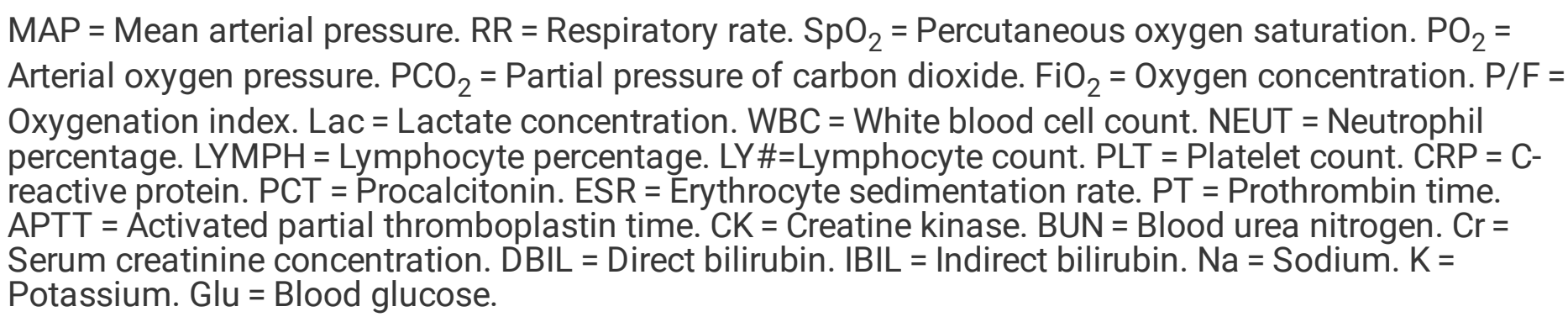 } \\
\hline
\end{tabular}




\begin{tabular}{|c|c|c|c|}
\hline & Critical type(n=15) & Severe type(n=22) & $\mathbf{P}$ \\
\hline $\operatorname{ESR}(\mathrm{mm} / \mathrm{h})$ & $52.50(47.00,81.50)$ & $33.00(13.25,49.75)$ & 0.03 \\
\hline PT (s) & $13.23(1.78)$ & $12.24(1.46)$ & 0.09 \\
\hline APTT (s) & $33.80(29.30,38.70)$ & $31.85(29.90,37.80)$ & 0.88 \\
\hline Troponin (ng/mL) & $0.02(0.01,0.15)$ & $0.02(0.01,0.03)$ & 0.49 \\
\hline CK (U/L) & $165.10(58.00,271.00)$ & $57.00(37.50,137.00)$ & 0.03 \\
\hline NTpro-BNP (pg/mL) & $536.30(50.00,4119.00)$ & $127.04(55.05,479.98)$ & 0.14 \\
\hline BUN (mmol/L) & $8.00(5.24,9.80)$ & $4.84(3.79,6.97)$ & 0.03 \\
\hline $\mathrm{Cr}(\mu \mathrm{mol} / \mathrm{L})$ & $70.00(59.00,115.30)$ & $69.00(58.88,84.25)$ & 0.36 \\
\hline Cystatin C(mg/L) & $2.06(1.60,3.14)$ & $1.64(1.17,2.05)$ & 0.18 \\
\hline $\mathrm{DBIL}(\mu \mathrm{mol} / \mathrm{L})$ & $7.28(4.57)$ & 4.94(3.59) & 0.09 \\
\hline IBIL $(\mu \mathrm{mol} / \mathrm{L})$ & $9.24(4.49)$ & $9.38(5.23)$ & 0.93 \\
\hline Albumin $(\mathrm{g} / \mathrm{L})$ & $33.19(4.14)$ & $36.09(5.36)$ & 0.08 \\
\hline $\mathrm{Na}(\mathrm{mmol} / \mathrm{L})$ & $136.69(4.58)$ & 135.34(3.02) & 0.29 \\
\hline $\mathrm{K}(\mathrm{mmol} / \mathrm{L})$ & $4.21(0.47)$ & $3.81(0.66)$ & 0.05 \\
\hline Glu (mmol/L) & $9.97(7.19,17.03)$ & $6.84(5.75,10.18)$ & 0.05 \\
\hline APACHE $\nabla$ score & 14.67(4.29) & $9.09(2.58)$ & 0.00 \\
\hline SOFA score & $5(4,10)$ & $3(2,3)$ & 0.00 \\
\hline \multicolumn{4}{|c|}{ Data are median(first quartile,third quartile)or mean(SD). } \\
\hline \multicolumn{4}{|c|}{ 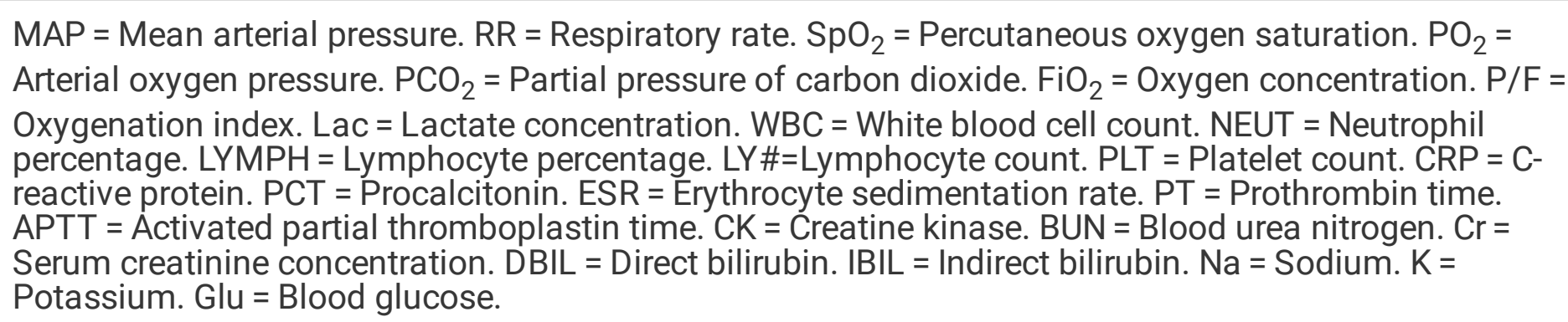 } \\
\hline
\end{tabular}


Table 4

Treatment and comorbidities of critical ill patients with COVID-19

\begin{tabular}{|c|c|c|c|c|}
\hline & $\begin{array}{l}\text { Critical type }(n= \\
15)\end{array}$ & Severe type $(n=22)$ & $\begin{array}{l}\text { All } \\
\text { patients(n } \\
=37)\end{array}$ & $\mathbf{P}$ \\
\hline Antiviral agents & $15(100 \%)$ & $22(100 \%)$ & $37(100 \%)$ & - \\
\hline Interferon & $14(93.30 \%)$ & $22(100 \%)$ & $36(97.30)$ & 0.22 \\
\hline Lopinavir/ritonavir & $14(93.30 \%)$ & $20(90.90 \%)$ & $34(91.90)$ & 0.79 \\
\hline Arbidol & $10(66.70 \%)$ & $16(72.70 \%)$ & $26(70.30 \%)$ & 0.69 \\
\hline Oseltamivir & $2(13.30 \%)$ & $4(18.20 \%)$ & $6(16.20 \%)$ & 0.69 \\
\hline $\begin{array}{l}\text { Antibacterial } \\
\text { agents }\end{array}$ & $15(100 \%)$ & $18(81.80 \%)$ & $33(89.20 \%)$ & 0.08 \\
\hline Moxifloxacin & $13(86.70 \%)$ & $13(59.10 \%)$ & $26(70.30 \%)$ & 0.07 \\
\hline Levofloxacin & $3(20.00 \%)$ & $5(22.70 \%)$ & $8(21.60 \%)$ & 0.84 \\
\hline TZP & $9(60.00 \%)$ & $5(22.70 \%)$ & $14(37.80 \%)$ & 0.02 \\
\hline SCF & $6(40.00 \%)$ & $2(9.10 \%)$ & $8(21.60 \%)$ & 0.03 \\
\hline Azithromycin & $1(6.70 \%)$ & $3(13.60 \%)$ & $4(10.80 \%)$ & 0.50 \\
\hline Imipenem & $3(20.00 \%)$ & $1(4.50 \%)$ & $4(10.80 \%)$ & 0.14 \\
\hline Meropenem & $2(13,30 \%)$ & 0 & $2(5.40 \%)$ & 0.08 \\
\hline Carbapenems & $5(33.30 \%)$ & $1(4.50 \%)$ & $6(16.20 \%)$ & 0.02 \\
\hline Vancomycin & $3(20.00 \%)$ & 0 & $3(8.10 \%)$ & 0.03 \\
\hline Linezolid & $2(13.30 \%)$ & 0 & $2(5.40 \%)$ & 0.08 \\
\hline Ceftriaxone & $1(6.70 \%)$ & $1(4.50 \%)$ & $2(5.40 \%)$ & 0.78 \\
\hline Antifungal agents & $4(26.70 \%)$ & 0 & $4(10.80 \%)$ & 0.01 \\
\hline Methylprednisolone & $15(100 \%)$ & $20(90.90 \%)$ & $35(94.60 \%)$ & 0.23 \\
\hline Hydrocortisone & $1(6.70 \%)$ & 0 & $1(2.70 \%)$ & 0.22 \\
\hline Gamma globulin & $10(66.70 \%)$ & $9(40.90 \%)$ & $19(51.40 \%)$ & 0.12 \\
\hline
\end{tabular}

Data are median (first quartile,third quartile) or mean (SD).

rhBNP = Lyophilized Recombinant Human Brain Natriuretic Peptide. TZP = Piperacillin Sodium and Tazobactam Sodium. SCF = Cefoperazone Sodium and Sulbactam Sodium/Tazobactam Sodium. CRRT $=$ Continuous renal replacement therapy. 


\begin{tabular}{|c|c|c|c|c|}
\hline & $\begin{array}{l}\text { Critical type }(\mathrm{n}= \\
\text { 15) }\end{array}$ & Severe type $(n=22)$ & $\begin{array}{l}\text { All } \\
\text { patients(n } \\
=37)\end{array}$ & $\mathbf{P}$ \\
\hline Human Albumin & 10(66.70\%) & $6(27.30 \%)$ & $16(43.20 \%)$ & 0.02 \\
\hline Blood products & $9(60.00 \%)$ & $1(4.50 \%)$ & $10(27.00 \%)$ & 0.00 \\
\hline Thymalfasin & $8(53.30 \%)$ & $8(36.40 \%)$ & $16(43.20 \%)$ & 0.31 \\
\hline Xuebijing injection & $7(46.70 \%)$ & $10(45.50 \%)$ & $17(45.90 \%)$ & 0.94 \\
\hline $\begin{array}{l}\text { Traditional Chinese } \\
\text { Drugs }\end{array}$ & 14(93.30\%) & $21(95.50 \%)$ & $35(94.60 \%)$ & 0.78 \\
\hline rhBNP & $4(26.70 \%)$ & 0 & $4(10.80 \%)$ & 0.01 \\
\hline Levosimendan & $3(20.00 \%)$ & 0 & $3(8.10 \%)$ & 0.03 \\
\hline Vasoactive agent & $9(60.00 \%)$ & 0 & $9(24.30 \%)$ & 0.00 \\
\hline Dopamine & $4(26.70 \%)$ & 0 & $4(10.80 \%)$ & 0.01 \\
\hline Noradrenaline & $9(60.00 \%)$ & 0 & $9(24.30 \%)$ & 0.00 \\
\hline Sedatives & $10(66.70 \%)$ & 0 & $10(27.00 \%)$ & 0.00 \\
\hline Propofol & $9(60.00 \%)$ & 0 & $9(24.30 \%)$ & 0.00 \\
\hline Dexmedetomidine & $5(33.30 \%)$ & 0 & $5(13.50 \%)$ & 0.00 \\
\hline Midazolam & $10(66.70 \%)$ & 0 & $10(27.00 \%)$ & 0.00 \\
\hline Analgesics & $10(66.70 \%)$ & 0 & $10(27.00 \%)$ & 0.00 \\
\hline fentanyl & $9(60.00 \%)$ & 0 & $9(24.30 \%)$ & 0.00 \\
\hline Dezocine & $1(6.70 \%)$ & 0 & $1(2.70 \%)$ & 0.22 \\
\hline $\begin{array}{l}\text { Neuromuscular } \\
\text { blockingagents }\end{array}$ & $4(26.70 \%)$ & 0 & $4(10.80 \%)$ & 0.01 \\
\hline Diuretics & 13(86.70\%) & $8(36.40 \%)$ & $21(56.80 \%)$ & 0.00 \\
\hline nasal cannula & 0 & $19(86.36 \%)$ & $\begin{array}{l}19 \\
(51.35 \%)\end{array}$ & 0.00 \\
\hline high-flow & $2(13.33 \%)$ & $3(13.64 \%)$ & $5(13.51 \%)$ & 1.00 \\
\hline invasive ventilation & $9(60.00 \%)$ & 0 & $9(24.32 \%)$ & 0.21 \\
\hline \multicolumn{5}{|c|}{ Data are median (first quartile,third quartile) or mean (SD). } \\
\hline \multicolumn{5}{|c|}{$\begin{array}{l}\text { rhBNP = Lyophilized Recombinant Human Brain Natriuretic Peptide. TZP = Piperacillin Sodium and } \\
\text { Tazobactam Sodium. SCF = Cefoperazone Sodium and Sulbactam Sodium/Tazobactam Sodium. } \\
\text { CRRT = Continuous renal replacement therapy. }\end{array}$} \\
\hline
\end{tabular}




\begin{tabular}{|c|c|c|c|c|}
\hline $\begin{array}{l}\text { noninvasive } \\
\text { ventilation }\end{array}$ & $4(26.67 \%)$ & 0 & $4(10.81 \%)$ & 0.00 \\
\hline $\begin{array}{l}\text { prone position } \\
\text { ventilation }\end{array}$ & $7(46.67 \%)$ & 0 & $7(18.92 \%)$ & 0.00 \\
\hline CRRT & $2(13.33 \%)$ & 0 & $2(5.41 \%)$ & 0.16 \\
\hline $\begin{array}{l}\text { Total liquid input in } \\
5 \text { days }(\mathrm{ml})\end{array}$ & $12684.00(10636.00,15011.85)$ & $\begin{array}{l}10120.00 \\
(7564.00,11251.00)\end{array}$ & $\begin{array}{l}11416.57 \\
(3157.91)\end{array}$ & 0.00 \\
\hline $\begin{array}{l}\text { Total liquid output } \\
\text { in } 5 \text { days }(\mathrm{ml})\end{array}$ & $13386.00(2781.52)$ & 9757.15 (3837.89) & $\begin{array}{l}1157.58 \\
(3769.30)\end{array}$ & 0.01 \\
\hline $\begin{array}{l}\text { Total liquid balance } \\
\text { in } 5 \text { days }(\mathrm{ml})\end{array}$ & $-392.71(2404.71)$ & $9.54(2000.10)$ & $\begin{array}{l}-199.04 \\
(2186.23)\end{array}$ & 0.64 \\
\hline $\begin{array}{l}\text { Daily liquid } \\
\text { balance(ml) }\end{array}$ & $-78.50(480.81)$ & 31.01(378.85) & $\begin{array}{l}-20.09 \\
(425.39)\end{array}$ & 0.49 \\
\hline Comorbidities & $9(60.00 \%)$ & 0 & $9(24.30 \%)$ & 0.00 \\
\hline Cardiac injury & $6(40.00 \%)$ & 0 & $6(16.20 \%)$ & 0.00 \\
\hline Acute kidney injury & $4(26.70 \%)$ & 0 & $4(10.80 \%)$ & 0.01 \\
\hline $\begin{array}{l}\text { Gastrointestinal } \\
\text { bleeding }\end{array}$ & $4(26.70 \%)$ & 0 & $4(10.80 \%)$ & 0.01 \\
\hline \multirow[t]{2}{*}{ Liver dysfunction } & $1(6.70 \%)$ & 0 & $1(2.70 \%)$ & 0.22 \\
\hline & $\begin{array}{l}\text { Severe } \\
\text { type }(n=4)\end{array}$ & $\mathbf{P}$ & & \\
\hline
\end{tabular}

Data are median (first quartile,third quartile) or mean (SD).

rhBNP = Lyophilized Recombinant Human Brain Natriuretic Peptide. TZP = Piperacillin Sodium and Tazobactam Sodium. SCF = Cefoperazone Sodium and Sulbactam Sodium/Tazobactam Sodium.

CRRT $=$ Continuous renal replacement therapy. 


\begin{tabular}{|c|c|c|c|c|}
\hline & $\begin{array}{l}\text { Critical type }(\mathrm{n}= \\
\text { 15) }\end{array}$ & Severe type $(n=$ & & $\begin{array}{l}\text { All } \\
\text { patients(n } \\
=37)\end{array}$ \\
\hline CD4cell count $(/ \mu \mathrm{L})$ & $\begin{array}{l}160.00(101.00 \\
208.00)\end{array}$ & $\begin{array}{l}233.00 \\
(187.00 \\
298.50)\end{array}$ & 0.06 & \\
\hline CD8cell count $(/ \mu \mathrm{L})$ & $\begin{array}{l}114.00 \\
(101.00,304.00)\end{array}$ & $\begin{array}{l}138.50 \\
(98.00,217.25)\end{array}$ & 1.00 & \\
\hline CD3cell count $(/ \mu \mathrm{L})$ & $\begin{array}{l}292.00(234.00 \\
638.00)\end{array}$ & $\begin{array}{l}437.50 \\
(366.75 \\
461.00)\end{array}$ & 0.73 & \\
\hline CD4/CD8 & $\begin{array}{l}1.09(0.52, \\
1.62)\end{array}$ & $\begin{array}{l}2.05(0.99, \\
2.42)\end{array}$ & 0.19 & \\
\hline \multicolumn{5}{|c|}{ Data are median (first quartile,third quartile) or mean (SD). } \\
\hline \multicolumn{5}{|c|}{$\begin{array}{l}\text { rhBNP = Lyophilized Recombinant Human Brain Natriuretic Peptide. TZP = Piperacillin Sodium and } \\
\text { Tazobactam Sodium. SCF = Cefoperazone Sodium and Sulbactam Sodium/Tazobactam Sodium. } \\
\text { CRRT = Continuous renal replacement therapy. }\end{array}$} \\
\hline
\end{tabular}

\section{Treatment And Comorbidities}

All patients were treated with antiviral agents, 36 (97.30\%) with interferon, 34 (91.9\%) with lopinavir/ritonavir, $26(70.30 \%)$ with arbidol and $6(16.20 \%)$ with oseltamivir. Antibacterial agents were given to $33(89.20 \%)$ patients, moxifloxacin use was $70.30 \%$, piperacillin sodium and tazobactam sodium were $37.80 \%$, levofloxacin, cefoperazone sodium and sulbactam sodium/Tazobactam Sodium, and azithromycin were used occasionally (Table 4). Several patients with critical type used more broadspectrum macrolide antibiotics, such as carbapenems, vancomycin, linezolid or antifungal agents (Table 4). 35 (95.60\%) patients received methylprednisolone and traditional Chinese drugs, about one-half of the patients received immunizing agents, albumin and xuebijing, about one quarter patients received blood products (Table 4).

All patients had acute respiratory distress syndrome (ARDS), in the critical group, the degree of dyspnea was more severe and oxygenation index was lower (Table 3). Nineteen $(86.36 \%)$ severe type patients were treated with nasal cannula, only three (13.64\%) received high-flow nasal cannula. Nine $(60.00 \%)$ critical type patients were treated with invasive mechanical ventilation, four $(26.67 \%)$ with noninvasive mechanical ventilation, two (13.33\%) with high-flow nasal cannula, seven $(46.67 \%)$ with prone position ventilation, and two (13.33\%) with renal replacement therapy (Table 4). The patients with invasive ventilation received sedations and analgesics, usually needed vasoconstrictors (Table 4). Compared with those of severe group, the patients in critical group were given more fluid and more diuretics (Table 4). 
Nine $(60.00 \%)$ patients with critical type had other organ damage, six $(40.00 \%)$ with cardiac injury, four (26.70\%) with acute kidney injury, four $(26.70 \%)$ with gastrointestinal bleeding, one $(6.70 \%)$ with liver dysfunction (Table 4).

\section{Discussion}

Up to 14 February 2020, there remain to be nearly 10,000 critical ill patients with COVID-19 in China, and the management of this group of patients becomes the heart of the matter. We hope to provide valuable reference for other colleagues by describing their epidemiological characteristics and treatment status.

We described the epidemiological features of COVID-19 among critical ill patients. Half the patients were men over 60 years old, which were similar to that found in Wuhan and Zhejiang province, including mild and critical patients $[4,7]$. According to our results, no patients in Hebei province had been exposed to the Huanan seafood market, however, familial clusters were present in nearly half of the critical ill patients, which was different with Wuhan but similar with Zhejiang. Critical group and severe group showed similar sex, age distributions as well as history of exposure, but those with underlying diseases were more likely to develop to critical type, this is similar to Chen's study [8]. As compared with critical ill patients in Wuhan, critical ill patients in Hebei province had a higher rate of chronic medicine disease, which may predict a worse prognosis [5]. Moreover, the length of the interval between symptom onset and diagnosis was longer

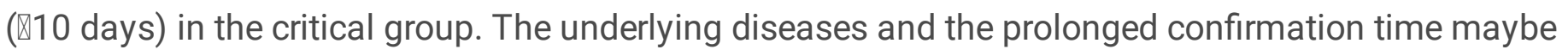
associated with the severity of the disease.

The cardinal clinical feature was fever, followed by cough, expectoration and dyspnea. Other symptoms include fatigue, myalgia, diarrhea and headache were not frequent. Cough and dyspnea were more common in patients with critical type. Chest CT changes are noticeable, most patients presented with bilateral ground-glass opacities, reticular pattern and consolidation. Lung damage was more severe in critical type than in severe type, which is also in line with the previous reports $[9,10]$. Pan et al $[9]$ found that in the early stage, one or two lobes were involved, the lung function remained normal. The consolidation area of the lung began to absorb and became a ground glass opacity or fiber cord focus as the disease progression. The ventilation function will be limited and contributed to disability.

All vital signs we recorded showed that patients in critical group had severe dyspnea and hypotension, and required stronger respiratory support or more vasoactive agents. Early studies have shown leucopenia and lymphopenia were common in general patients with COVID-19 [7, 8]. However, Huang et al [8] reported that white blood cell count and levels of inflammatory cytokines were higher in ICU patients than non-ICU patients, indicating that the cytokine storm was related to the severity of the illness. A study of autopsy [11]found that a marked reduction of the counts of peripheral CD4 and CD8 in the critical patient with COVID-19. Our data from laboratory findings gave similar results, critical type patients had higher levels of WBC, NEUT, ESR, CRP and PCT than severe type patients, but LYMPH, T cell subsets and oxygenation index were lower, suggesting stronger inflammatory responses, and severe lung injury. As a result, most patients in critical group had complications and received more broad-spectrum antibiotic or antifungal therapy. Additionally, patients with critical type had mild elevation of creatine kinase. The biomarkers of 
coagulation, liver and kidney function, electrolytes were within normal range, but the patients in critical group received more immunizing agents, albumin and blood products. Patients with critical type also had higher APACHE II, SOFA scores and complications, specifically of heart and kidney, which was in keeping with the results of previous studies [7, 8].

Antiviral agents, including interferon, lopinavir/ritonavir and arbidol, were given to all patients, with a few of them also received oseltamivir. The lopinavir/ritonavir is recommended in the Guidelines for Diagnosis and Treatment of Pneumonia Caused by 2019-nCoV (version 5), and arbidol is used as antiviral treatment in Russia and China for influenza infection [12]. Previous clinical studies evaluated the effect of interferon in severe acute respiratory syndrome (SARS) and Middle East respiratory syndrome (MERS), but benefits from interferon and antiviral drugs are still in discussing $[13,14]$.Traditional Chinese drugs had been widely used, and received good results in mild patients [15]. However, its value in critically ill patients has not been well understood. Fifteen (100\%) critical type patients and 22 (90.90\%) severe type patients received lowdose methylprednisolone, which was also a challenging medication. World Health Organization (WHO) recommends against the routine use of corticosteroids for treatment of ARDS or shock induced by COVID19 pneumonia, because no clinical evidence to supports the benefits from corticosteroids in the treatment of SARS, MERS or influenza [16]. In China, combinations of low-dose and short-course methylprednisolone with immunosuppressive drugs are recommended to critical ill patients infected by COVID-19, further investigation needs to be performed in this area.

The pathogenesis of COVID-19 pneumonia is not well established. Like SARS and MERS, severe ARDS is the result of pathophysiologic processes underlying severe viral pneumonia $[5,17-20]$. The severity of ARDS also varied among critical ill patients with COVID-19 pneumonia in Hebei province. Most critical type patients were in the stage of severe ARDS, and majority severe type patients were in the period of mild ARDS. Unfortunately, despite studied for decades, the treatment of severe ARDS remains supportive with lung-protective mechanical ventilation, such as low tidal-volume mechanical ventilation, prone position and extracorporeal membrane oxygenation (ECMO) [21, 22]. In our study, just $60 \%$ in critical type patients were treated with invasive mechanical ventilation, less than half of these patients received prone position, no patient received ECMO. We did not find detailed ventilator parameters in the electronic medical records. Blood gases analysis revealed a lower level of plasma $\mathrm{PCO}_{2}$, implying an unsatisfactory results of ventilator settings, sedation or analgesia. Fluid management is also a key point in ARDS. Compared with liberal fluid management, conservative fluid management could significantly decrease the degree of lung edema, the length of ventilator days, the length of ICU stay, and mortality for patients with ARDS [23, 24]. Our results showed that critical group received more fluid and more diuretics, suggesting the physicians may be aware of these guidelines but unable to follow the rules because of the severity of the illness. Some problems remain uninvestigated because the majority of patients were still receiving treatment. Further research is needed to investigate the risk factors associated with the prognosis.

There are several potential weaknesses of our retrospective analysis. First, the number of patients in this study was small, higher missing data rates are present in parameters. Second, these cases in Hebei province represented a rather small part of the critical ill patients with COVID-19 pneumonia, needing to be 
further evaluation in other regions. Third, at the time of data collection, most patients were still on treatment, we were unable to evaluate their prognosis.

\section{Conclusion}

All together,we should pay more attention to the patients aged over 60 years who had underlying disease to high risk infected. Vital signs, circulating levels of inflammatory markers, radiologic imaging and blood gas analysis must be monitored carefully, early detection and management of critical ill patients are crucial for a favorable outcome. We still face the fact that, there is no evidence-based treatment strategy because the pathophysiology of this disease is uncertain, most critical ill patients were treated with supportive therapy. Patients with severe ARDS should strictly follow the strategy of lung-protective mechanical ventilation and restrictive fluid management.

\section{Declarations}

\section{Abbreviations}

COVID-19: Novel coronavirus disease 2019; $\mathrm{SpO}_{2}$ : Percutaneous oxygen saturation; $\mathrm{PaO}_{2}: \mathrm{Partial}$ pressure of oxygen; FiO2: Fraction of inspired oxygen; MAP: mean arterial pressure; RR: respiratory rate; CT: Chest computed tomography; Lac: Lactate concentration; WBC: White blood cell count; NEUT: Neutrophil percentage; LYMPH: Lymphocyte percentage; LY\#: Lymphocyte count; PLT: Platelet count; CRP: C-reactive protein; PCT: Procalcitonin; ESR: Erythrocyte sedimentation rate; PT: Prothrombin time; APTT: Activated partial thromboplastin time; CK: Creatine kinase; BUN: Blood urea nitrogen; Cr: Creatinine concentration; DBIL: Direct bilirubin; IBIL: Indirect bilirubin; Na: Sodium; K: Potassium; Glu: Glucose; APACHE 囚: Acute physiology and chronic health evaluation II; SOFA: Sequential organ failure assessment; rhBNP: Recombinant human brain natriuretic peptide; SD: Standard deviation; ARDS: Acute respiratory distress syndrome; SARS: Severe acute respiratory syndrome; WHO: World health organization; ECMO:

Extracorporeal membrane oxygenation

\section{Ethics approval and consent to participate}

The Ethics Commission of the Fourth Clinical Medical College of Hebei Medical University approved this study(2020KS002).

\section{Consent for publication}

Not applicable

\section{Availability of data and materials}

After publication, we could share the data to other researchers, and a statement will be needed for evaluating the reasonability and validity. With agreement of the corresponding author and designated hospitals to treat patients with COVID-19, raw data will be provided. 


\section{Competing interests}

The authors declare that there are no conflicts of interests regarding the publication of this paper.

\section{Funding}

This study was supported by the Department of Science and Technology of Hebei Province of China (20277707D).

\section{Authors' contributions}

Yuhong Chen and Zhenjie Hu were involved in design. Kun Zhang®Yuhong Chen『Zhigang Cai and Lixia Liu collected the epidemiological and clinical data. Kun Zhang, Yuhong Chen and Zhongheng Zhang summarized the data and performed analysis. Yuhong Chen drafted the manuscript. Xixin Yan, Guijun Zhu and Zhenjie Hu revised the final manuscript.

\section{Acknowledgements}

Authors wish to acknowledge all patients and their families involved in this study. We are grateful to the Expert Panel of the COVID-19 of Hebei province and all health care workers around the word. We also thank Health Care Committee of Hebei province for data support, and the Department of Science and Technology of Hebei province for financial support.

\section{Authors' information}

${ }^{1}$ Department of Critical Care Medicine, Hebei Medical University Fourth Affiliated Hospital and Hebei Provincial Tumor Hospital, 12 jiankang Road Shijiazhuang, Hebei, 050011, China

${ }^{2}$ Department of Respiration, Hebei Medical University Second Affiliated Hospital, 215 heping Road Shijiazhuang, Hebei, 050011, China

${ }^{3}$ Department of Emergency Medicine, Sir Run Run Shaw Hospital, Zhejiang University School of Medicine, 3 qingchun road, Hangzhou, 310016, China,

*Correspondence Author: Prof. Zhenjie Hu, Tel: +086 03118609 5367, syicu@vip.sina.com

Back-up Author: Yuhong Chen, yuhong_apple@126.com.

\section{References}

1. Special Expert Group for Control of the Epidemic of Novel Coronavirus Pneumonia of the Chinese Preventive Medicine Association. [An update on the epidemiological characteristics of novel coronavirus pneumonia『COVID-19邓]. Zhonghua Liu Xing Bing Xue Za Zhi. 2020;41:139-44.

2. Wang D, Hu B, Hu C, Zhu F, Liu X, Zhang J, et al. Clinical Characteristics of 138 Hospitalized Patients With 2019 Novel Coronavirus-Infected Pneumonia in Wuhan, China. JAMA. 2020. 
3. Diagnosis and Treatment of Pneumonia Caused by 2019-nCoV (version 5). http://www.nhc.gov.cn/yzygj/s7653p/202002/3b09b894ac9b4204a79db5b8912d4440.shtml (accessed February 5, 2020). (in Chinese) .

4. Xu XW, Wu XX, Jiang XG, Xu KJ, Ying LJ, Ma CL, et al. Clinical findings in a group of patients infected with the 2019 novel coronavirus (SARS-Cov-2) outside of Wuhan, China: retrospective case series. BMJ. 2020;368:m606.

5. Yang X, Yu Y, Xu J, Shu H, Xia J, Liu H, et al. Clinical course and outcomes of critically ill patients with SARS-CoV-2 pneumonia in Wuhan, China: a single-centered, retrospective, observational study. Lancet Respir Med. 2020.

6. Zhang Z. Univariate description and bivariate statistical inference: the first step delving into data. Ann Transl Med. 2016;4:91.

7. Zhang JJ, Dong X, Cao YY, Yuan YD, Yang YB, Yan YQ, et al. Clinical characteristics of 140 patients infected with SARS-CoV-2 in Wuhan, China. Allergy. 2020.

8. Huang C, Wang Y, Li X, Ren L, Zhao J, Hu Y, et al. Clinical features of patients infected with 2019 novel coronavirus in Wuhan, China. Lancet. 2020;395:497-506.

9. Pan Y, Guan H. Imaging changes in patients with 2019-nCov. Eur Radiol. 2020.

10. Pan Y, Guan H, Zhou S, Wang Y, Li Q, Zhu T, et al. Initial CT findings and temporal changes in patients with the novel coronavirus pneumonia (2019-nCoV): a study of 63 patients in Wuhan, China. Eur Radiol. 2020.

11. Xu Z, Shi L, Wang Y, Zhang J, Huang L, Zhang C, et al. Pathological findings of COVID-19 associated with acute respiratory distress syndrome. Lancet Respir Med. 2020.

12. Wang Y, Ding Y, Yang C, Li R, Du Q, Hao Y, et al. Inhibition of the infectivity and inflammatory response of influenza virus by Arbidol hydrochloride in vitro and in vivo (mice and ferret). Biomed Pharmacother. 2017;91:393-401.

13. Lau AC, Yam LY, So LK. Management of Critically III Patients with Severe Acute Respiratory Syndrome (SARS). Int J Med Sci. 2004;1:1-10.

14. Arabi YM, Shalhoub S, Mandourah Y, Al-Hameed F, Al-Omari A, Al Qasim E, et al. Ribavirin and Interferon Therapy for Critically III Patients With Middle East Respiratory Syndrome: A Multicenter Observational Study. Clin Infect Dis. 2019.

15. Wang Z, Chen X, Lu Y, Chen F, Zhang W. Clinical characteristics and therapeutic procedure for four cases with 2019 novel coronavirus pneumonia receiving combined Chinese and Western medicine treatment. Biosci Trends. 2020.

16. Russell CD, Millar JE, Baillie JK. Clinical evidence does not support corticosteroid treatment for 2019nCoV lung injury. Lancet. 2020;395:473-5.

17. Arabi YM, Al-Omari A, Mandourah Y, Al-Hameed F, Sindi AA, Alraddadi B, et al. Critically III Patients With the Middle East Respiratory Syndrome: A Multicenter Retrospective Cohort Study. Crit Care Med. 2017;45:1683-95. 
18. Arabi YM, Arifi AA, Balkhy HH, Najm H, Aldawood AS, Ghabashi A, et al. Clinical course and outcomes of critically ill patients with Middle East respiratory syndrome coronavirus infection. Ann Intern Med. 2014;160:389-97.

19. Kalil AC, Thomas PG. Influenza virus-related critical illness: pathophysiology and epidemiology. Crit Care. 2019;23:258.

20. Lew TW, Kwek TK, Tai D, Earnest A, Loo S, Singh K, et al. Acute respiratory distress syndrome in critically ill patients with severe acute respiratory syndrome. JAMA. 2003;290:374-80.

21. Fan E, Brodie D, Slutsky AS. Acute Respiratory Distress Syndrome: Advances in Diagnosis and Treatment. JAMA. 2018;319:698-710.

22. Qiu HB, Li XY, Du B, Kang H, Wang YS, Wang F, et al. [The keypoints in treatment of the critical novel coronavirus pneumonia patient]. Zhonghua Jie He He Hu Xi Za Zhi. 2020;43:E022.

23. Warren MA, Zhao Z, Koyama T, Bastarache JA, Shaver CM, Semler MW, et al. Severity scoring of lung oedema on the chest radiograph is associated with clinical outcomes in ARDS. Thorax. 2018;73:8406.

24. Silversides JA, Major E, Ferguson AJ, Mann EE, McAuley DF, Marshall JC, et al. Conservative fluid management or deresuscitation for patients with sepsis or acute respiratory distress syndrome following the resuscitation phase of critical illness: a systematic review and meta-analysis. Intensive Care Med. 2017;43:155-70.

\section{Supplementary Files Legend}

Supplement Table $1 \mathrm{~T}$ Cell Subsets of critical ill patients with COVID-19

\section{Figures}


44 critical ill patients with COVID-19 pneumonia from Jan 22, 2020 to Feb 14, 2020 included

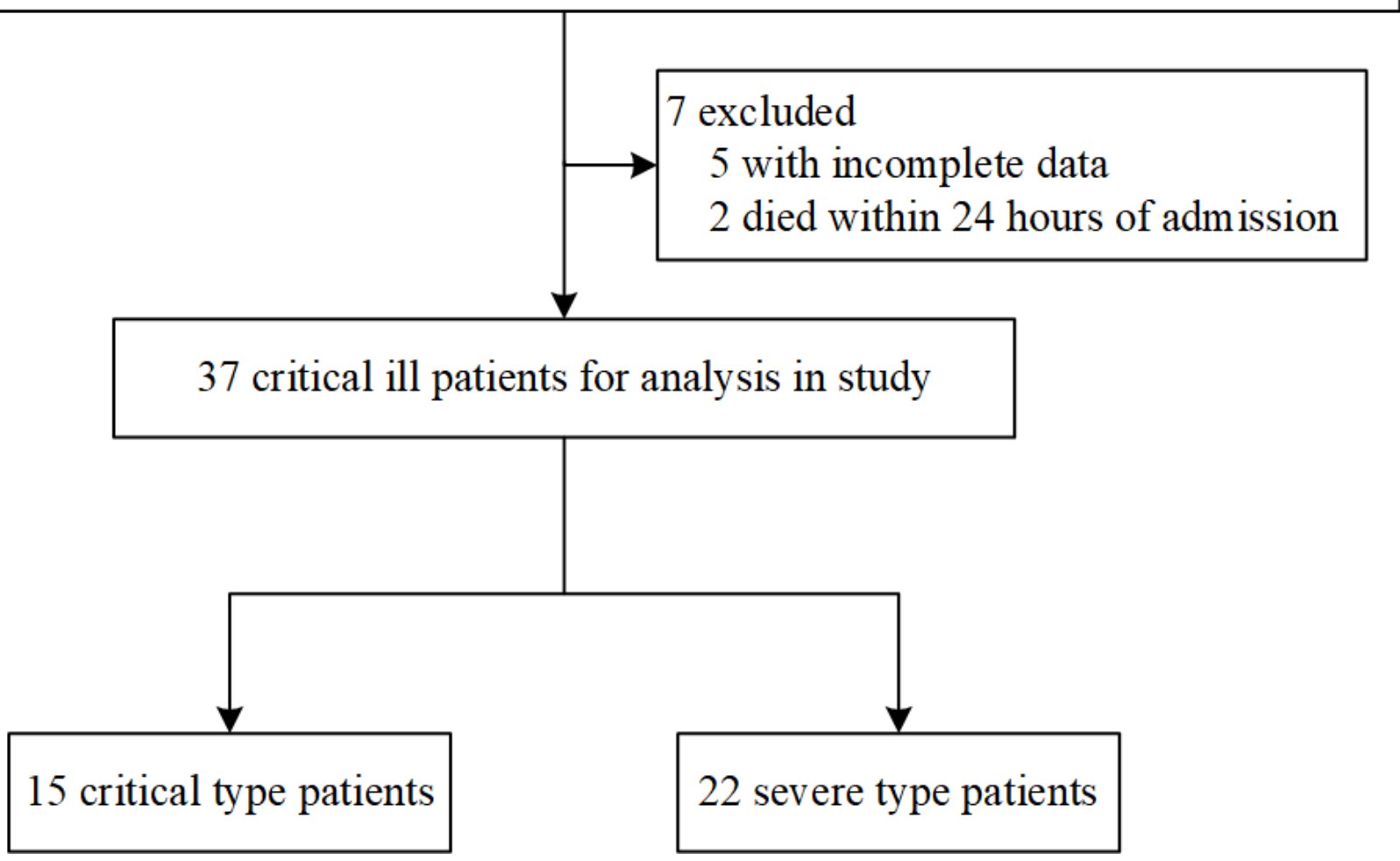

Figure 1

Study flow diagram 


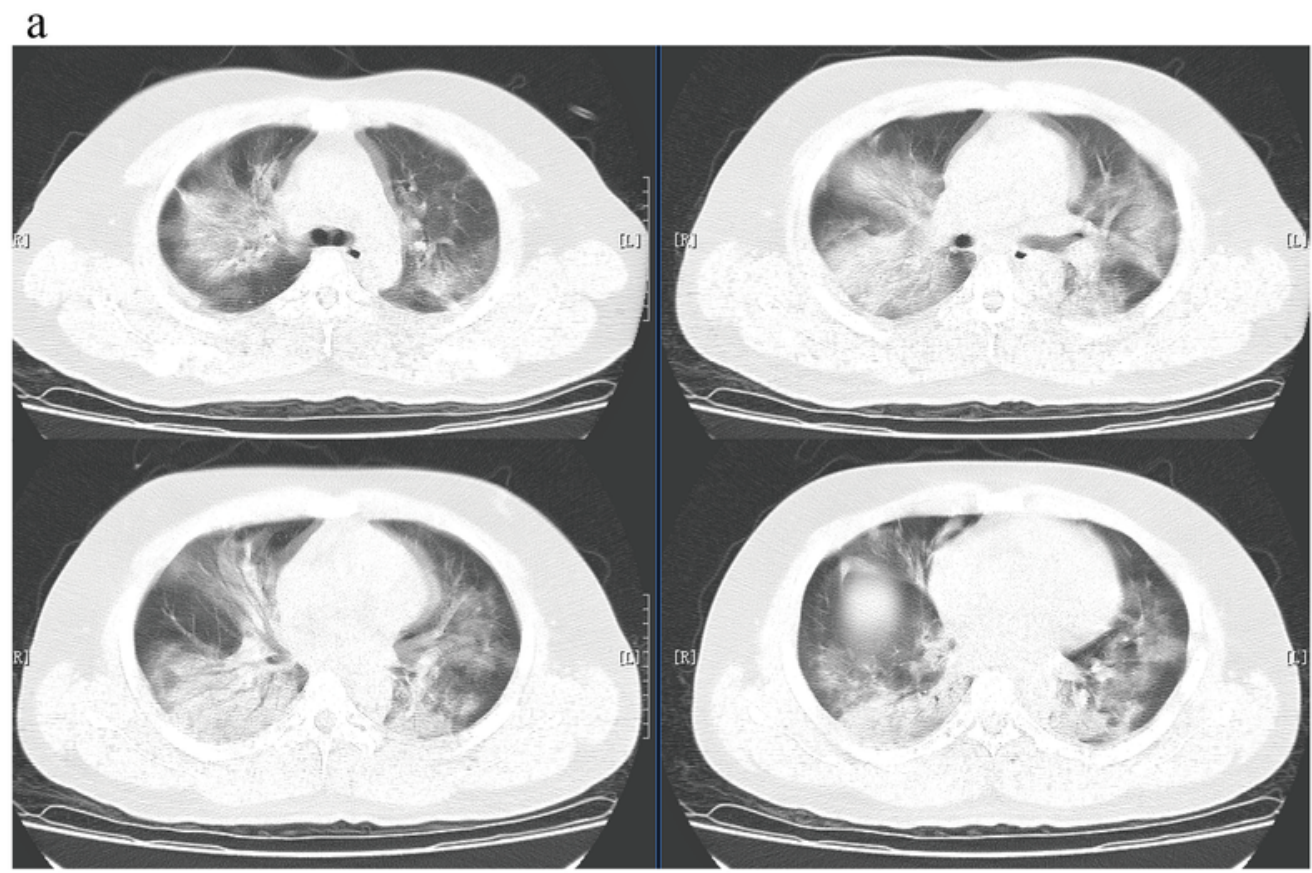

$\mathrm{b}$

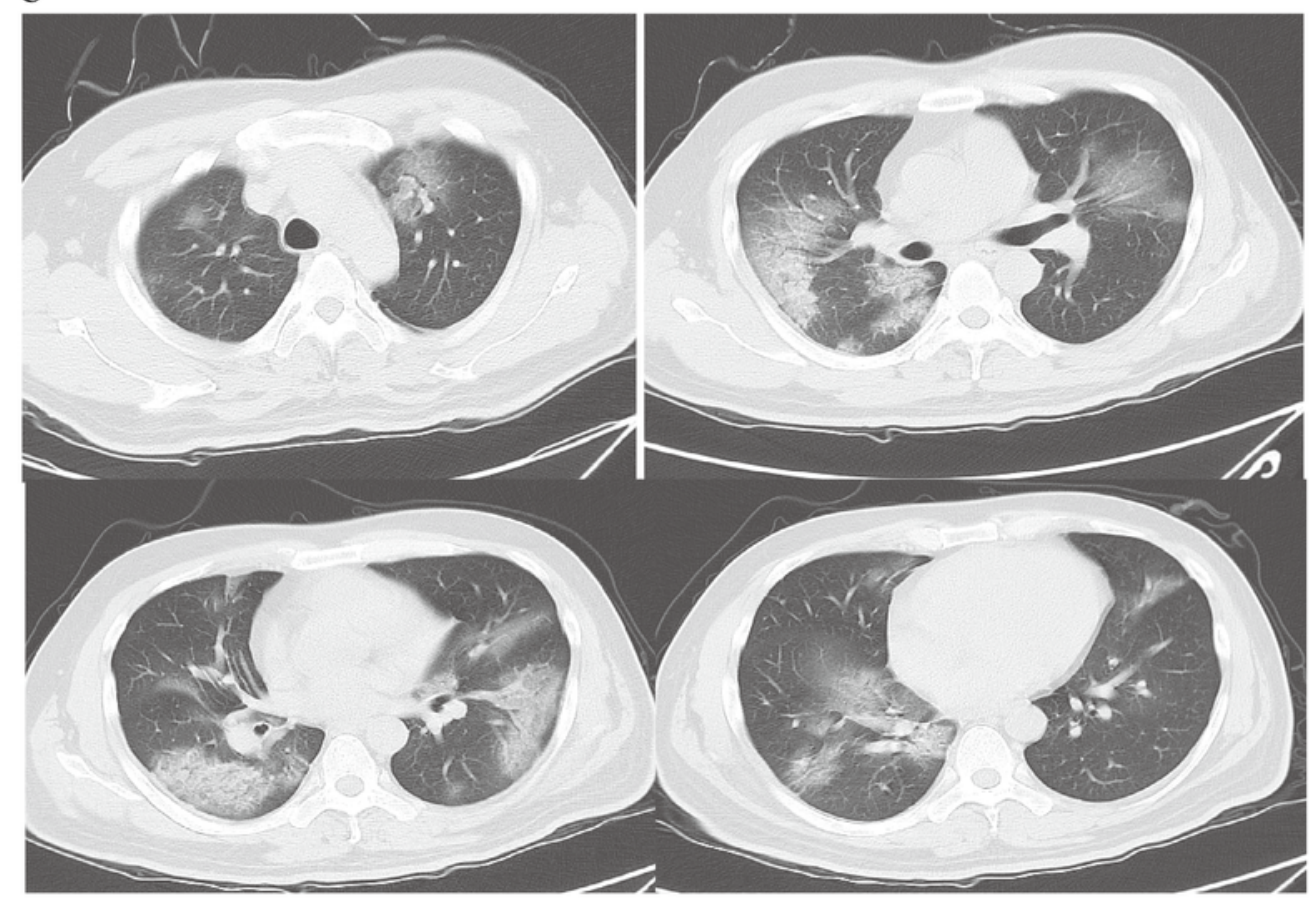

Figure 2

Chest computed tomography of critical ill patients (a) In most patients with critical type, chest computed tomogram scans demonstrated bilateral diffuse ground-glass opacities, reticular pattern and consolidation. (b) In most patients with severe type, the main patterns of abnormality on chest computed tomogram consist of localized ground glass opacity of both lungs. 


\section{Supplementary Files}

This is a list of supplementary files associated with this preprint. Click to download.

- SupplementTable1TCellSubsetsofcriticalillpatientswithCOVID.docx 